\title{
EVALUATION OF CLASSIFICATION ALGORITHMS USING MCDM AND RANK CORRELATION*
}

\author{
GANG KOU ${ }^{\dagger}$, YANQUN LU $^{\dagger}$, YI PENG ${ }^{\dagger}, \pi$ and YONG SH${ }^{\dagger}, \S$ \\ †School of Management and Economics \\ University of Electronic Science and Technology of China \\ Chengdu, 610054, P. R. China \\ ¿College of Information Science \& Technology \\ University of Nebraska at Omaha \\ Omaha, NE 68182, USA \\ $\S_{C A S}$ Research Center on Fictitious Economy \\ and Data Sciences, Beijing 100080, China \\ "pengyicd@gmail.com
}

\begin{abstract}
Classification algorithm selection is an important issue in many disciplines. Since it normally involves more than one criterion, the task of algorithm selection can be modeled as multiple criteria decision making (MCDM) problems. Different MCDM methods evaluate classifiers from different aspects and thus they may produce divergent rankings of classifiers. The goal of this paper is to propose an approach to resolve disagreements among MCDM methods based on Spearman's rank correlation coefficient. Five MCDM methods are examined using 17 classification algorithms and 10 performance criteria over 11 public-domain binary classification datasets in the experimental study. The rankings of classifiers are quite different at first. After applying the proposed approach, the differences among MCDM rankings are largely reduced. The experimental results prove that the proposed approach can resolve conflicting MCDM rankings and reach an agreement among different MCDM methods.
\end{abstract}

Keywords: Multi-criteria decision making (MCDM); classification; Spearman's rank correlation coefficient; TOPSIS; ELECTRE; grey relational analysis; VIKOR; PROMETHEE.

\section{Introduction}

As one of the most important tasks in data mining and knowledge discovery (DMKD) ${ }^{31}$ classification has been extensively studied and various algorithms have been developed over the years. Classification algorithms evaluation and selection is an active research area in the fields of machine learning, statistics, computer science, and DMKD. Rice ${ }^{37}$ formalized algorithm selection as abstract models with the problem space, the feature space, the criteria space, the algorithm space, and the

*Authors contributed equally to this work and are alphabetically ordered by their last names.

This is an Open Access article published by World Scientific Publishing Company. It is distributed under the terms of the Creative Commons Attribution 4.0 (CC-BY) License. Further distribution of this work is permitted, provided the original work is properly cited. 
performance measures. Nakhaeizadeh and Schnabl ${ }^{26}$ suggested multi-criteria-based metrics to compare classification algorithms. Smith-Miles ${ }^{42}$ treated the algorithm selection problem as a learning task and presented a unified framework that combines the cross-disciplinary developments in the algorithm selection problem. Peng et al. ${ }^{35}$ applied multiple criteria decision making (MCDM) methods to rank classification algorithms and validated the approaches using software defect datasets.

The evaluation of classification algorithms normally involves two or more conflicting criteria, such as accuracy, Type-I error, Type-II error, AUC, and computation time. Thus it can be modeled as a MCDM problem. ${ }^{41}$ Many MCDM methods have been developed and applied in a wide variety of applications. ${ }^{7,8,14,19,20,23,32,48}$ MCDM methods rank alternatives using different approaches. Applying various MCDM methods to a sorting problem is beneficial because the ranking agreed by several MCDM methods is more trustful than one generated by single MCDM method. Though some studies shown that MCDM methods provide similar rankings of alternatives, ${ }^{33,35}$ there are situations where different MCDM methods produce conflicting rankings. How to reconcile these differences becomes an important issue and has not been fully investigated. ${ }^{34}$

This paper combines MCDM methods with Spearman's rank correlation coefficient to rank classification algorithms. This approach first uses several MCDM methods to rank classification algorithms and then applies Spearman's rank correlation coefficient to resolve differences among MCDM methods. Five MCDM methods, including TOPSIS, ELECTRE III, grey relational analysis, VIKOR, and PROMETHEE II are implemented in this research. An experimental study, which chooses a wide selection of classifiers and performance measures, is conducted to validate the proposed approach over 11 public-domain binary classification datasets.

The rest of this paper is organized as follows. In Sec. 2, the proposed approach, including the selected MCDM methods and Spearman's rank correlation coefficient, is described. Section 3 presents the datasets, classification algorithms, performance measures, and the design of the experimental study. The results are discussed in Sec. 4. The final section summarizes the papers.

\section{Research Methodology}

Many MCDM methods have been developed to rank alternatives in different ways. While the rankings of alternatives provided by MCDM methods may in agreement sometimes, there are situations where different MCDM methods generate very different rankings. This work proposes to use Spearman's rank correlation coefficient to generate the final ranking that resolves or reduces differences among MCDM rankings. The proposed approach uses several MCDM methods to rank classifiers first; then applies Spearman's rank correlation coefficient to determine weights for MCDM methods to get secondary rankings of classifiers. This section describes the five MCDM methods used in the study and explains the details of the proposed approach. 


\subsection{MCDM methods}

Ranking classification algorithms normally need to examine multiple criteria, such as accuracy, AUC, F-measure, and kappa statistic. Therefore, the task of algorithm selection can be modeled as MCDM problems. This section gives an overview of the five selected MCDM methods (i.e., TOPSIS, ELECTRE III, grey relational analysis, VIKOR, and PROMETHEE II).

\section{Technique for Order Preference by Similarity to Ideal Solution (TOPSIS)}

Hwang and Yoon ${ }^{15}$ proposed the technique for order preference by similarity to ideal solution (TOPSIS) method to rank alternatives over multiple criteria. It finds the best alternatives by minimizing the distance to the ideal solution and maximizing the distance to the nadir or negative-ideal solution. ${ }^{27}$

A number of extensions and variations of TOPSIS have been developed over the years. The following TOPSIS procedure adopted from Opricovic and Tzeng ${ }^{29}$ and Olson $^{27}$ is used in the empirical study:

Step 1. Calculate the normalized decision matrix. The normalized value $r_{i j}$ is calculated as

$$
r_{i j}=x_{i j} / \sqrt{\sum_{j=1}^{J}} x_{i j}^{2}, \quad j=1, \ldots, J ; i=1, \ldots, n
$$

where $J$ and $n$ denote the number of alternatives and the number of criteria, respectively. For alternative $A_{j}$, the performance measure of the $i$ th criterion $C_{i}$ is represented by $x_{i j}$.

Step 2. Develop a set of weights $w_{i}$ for each criterion and calculate the weighted normalized decision matrix. The weighted normalized value $v_{i j}$ is calculated as

$$
v_{i j}=w_{i} x_{i j}, \quad j=1, \ldots, J ; \quad i=1, \ldots, n,
$$

where $w_{i}$ is the weight of the $i$ th criterion, and $\sum_{i=1}^{n} w_{i}=1$.

Step 3. Find the ideal alternative solution $S^{+}$, which is calculated as

$$
S^{+}=\left\{v_{1}^{+}, \ldots, v_{n}^{+}\right\}=\left\{\left(\max _{j} v_{i j} \mid i \in I^{\prime}\right),\left(\min _{j} v_{i j} \mid i \in I^{\prime \prime}\right)\right\},
$$

where $I^{\prime}$ is associated with benefit criteria and $I^{\prime \prime}$ is associated with cost criteria.

Step 4. Find the negative-ideal alternative solution $S^{-}$, which is calculated as

$$
S^{-}=\left\{v_{1}^{-}, \ldots, v_{n}^{-}\right\}=\left\{\left(\min _{j} v_{i j} \mid i \in I^{\prime}\right),\left(\max _{j} v_{i j} \mid i \in I^{\prime \prime}\right)\right\} .
$$


Step 5. Calculate the separation measures, using the $n$-dimensional Euclidean distance. The separation of each alternative from the ideal solution is calculated as

$$
D_{j}^{+}=\sqrt{\sum_{i=1}^{n}\left(v_{i j}-v_{i}^{+}\right)^{2}}, \quad j=1, \ldots, J .
$$

The separation of each alternative from the negative-ideal solution is calculated as

$$
D_{j}^{-}=\sqrt{\sum_{i=1}^{n}\left(v_{i j}-v_{i}^{-}\right)^{2}}, \quad j=1, \ldots, J .
$$

Step 6. Calculate a ratio $R_{j}^{+}$that measures the relative closeness to the ideal solution and is calculated as

$$
R_{j}^{+}=D_{j}^{-} /\left(D_{j}^{+}+D_{j}^{-}\right), \quad j=1, \ldots, J .
$$

Step 7. Rank alternatives by maximizing the ratio $R_{j}^{+}$.

\section{ELimination and Choice Expressing REality (ELECTRE)}

ELECTRE stands for ELimination Et Choix Traduisant la REalité (ELimination and Choice Expressing the REality) and was first proposed by Roy ${ }^{38}$ to choose the best alternative from a collection of alternatives. Over the last four decades, a family of ELECTRE methods has been developed, including ELECTRE I, ELECTRE II, ELECTRE III, ELECTRE IV, ELECTRE IS, and ELECTRE TRI.

There are two main steps of ELECTRE methods: the first step is the construction of one or several outranking relations; the second step is an exploitation procedure that identifies the best compromise alternative based on the outranking relation obtained in the first step. ${ }^{11}$ ELECTRE III is chosen in this paper because it is appropriate for the sorting problem. The procedure can be summarized as follows ${ }^{24,39,40}$ :

Step 1. Define a concordance and discordance index set for each pair of alternatives $A_{j}$ and $A_{k}, j, k=1, \ldots, m ; i \neq k$.

Step 2. Add all the indices of an alternative to get its global concordance index $C_{k i}$.

Step 3. Define an outranking credibility degree $\sigma_{s}\left(A_{i}, A_{k}\right)$ by combining the discordance indices and the global concordance index.

Step 4. Define two outranking relations using descending and ascending distillation. Descending distillation selects the best alternative first and the worst alternative last. Ascending distillation selects the worst alternative first and the best alternative last.

Step 5. Alternatives are ranked based on ascending and descending distillation processes. 


\section{Grey Relational Analysis (GRA)}

Grey relational analysis (GRA) is a part of grey theory, which has been proposed to handle imprecise and incomplete information in grey systems, ${ }^{5}$ and has been proven to be suitable for selecting a best alternative. The main procedure of GRA includes generating the grey relation, defining the reference sequence, and calculating the grey relational coefficient and grey relational grade (Kuo et al., 2008). The first step normalizes performance values for every alternative to get a comparability sequence. The second step defines an ideal target sequence. The third step compares the comparability sequences with the ideal sequence and the last step calculates the grey relational grade to rank alternatives. This study adopts the procedure provided by Kuo et $a l .^{22}$ :

Step 1. Generate the grey relation:

Suppose there are $m$ alternatives and $n$ attributes, the $i$ th alternative is defined as $Y_{i}=\left\{y_{i 1}, y_{i 2}, \ldots, y_{i n}\right\} . y_{i j}$ is the performance value of attribute $j$ of alternative $i$. Using one of the following three equations, the values of $Y_{i}$ can be translated into the comparability sequence $X_{i}=\left\{x_{i 1}, x_{i 2}, \ldots, x_{i n}\right\}$ :

$$
\begin{aligned}
& x_{i j}=\frac{y_{i j}-\operatorname{Min}\left\{y_{i j}, i=1,2, \ldots, m\right\}}{\operatorname{Max}\left\{y_{i j}, i=1,2, \ldots, m\right\}-\operatorname{Min}\left\{y_{i j}, i=1,2, \ldots, m\right\}} \\
& \text { for } i=1,2, \ldots, m ; j=1,2, \ldots, n, \\
& x_{i j}=\frac{\operatorname{Max}\left\{y_{i j}, i=1,2, \ldots, m\right\}-y_{i j}}{\operatorname{Max}\left\{y_{i j}, i=1,2, \ldots, m\right\}-\operatorname{Min}\left\{y_{i j}, i=1,2, \ldots, m\right\}} \\
& \text { for } i=1,2, \ldots, m ; j=1,2, \ldots, n, \\
& x_{i j}=1-\frac{\left|y_{i j}-y_{j}^{*}\right| y_{i j}-\operatorname{Min}\left\{y_{i j}, i=1,2, \ldots, m\right\}}{\operatorname{Max}\left\{\operatorname{Max}\left\{y_{i j}, i=1,2, \ldots, m\right\}-y_{i j}^{*}, y_{i j}^{*}-\operatorname{Min}\left\{y_{i j}, i=1,2, \ldots, m\right\}\right\}} \\
& \text { for } i=1,2, \ldots, m ; j=1,2, \ldots, n,
\end{aligned}
$$

where $y_{j}^{*}$ represents the desired value. Equation (2.1) is used for the-larger-thebetter attributes, Eq. (2.2) is used for the-smaller-the-better attributes, and Eq. (2.3) is used for the-closer-the-desired-value- $y_{j}^{*}$-the-better.

Step 2. Define the reference sequence:

The performance values calculated from Step 1 are ranged from 0 to 1 . The ideal target sequence, also called the reference sequence, is defined as $X_{0}=\left\{x_{01}, x_{02}, \ldots, x_{0 n}\right\}=\{1,1, \ldots, 1\}$. The objective of GRA is to find an alternative that has the closest comparability sequence to the reference sequence.

Step 3. Calculate the grey relational coefficient using the following equation:

$$
\gamma\left(x_{0 j}, x_{i j}\right)=\frac{V_{\min }+\zeta V_{\max }}{V_{i j}+\zeta V_{\max }}, \quad i=1,2, \ldots, m ; j=1, \ldots, n,
$$


where $\zeta$ is the distinguishing coefficient, $\zeta \in[0,1]$. The distinguishing coefficient is used to expand or compress the range of the grey relational coefficient and it is defined as 0.5 in this study. $x_{0 j}$ is the reference sequence and defined as $\left(x_{01}, x_{01}, \ldots, x_{0 J}\right)=(1,1, \ldots, 1) . \gamma\left(x_{0 j}, x_{i j}\right)$ is the grey relational coefficient between $x_{0 j}$ and $x_{i j}$, and

$$
\begin{aligned}
V_{i j} & =\left|x_{0 j}-x_{i j}\right|, \\
V_{\min } & =\operatorname{Min}\left\{V_{i j}, i=1,2, \ldots, m ; j=1,2, \ldots, n\right\}, \\
V_{\max } & =\operatorname{Max}\left\{V_{i j}, i=1,2, \ldots, m ; j=1,2, \ldots, n\right\} .
\end{aligned}
$$

Step 4. Calculate the grey relational grade using the following equation:

$$
\Gamma\left(X_{0}, X_{i}\right)=\sum_{j=1}^{n} w_{j} \gamma\left(x_{0 j}, x_{i j}\right), \quad \text { for } i=1, \ldots, m,
$$

where $w_{j}$ is the weight of attribute $j$ and $\sum_{j=1}^{n} w_{i}=1$. The grey relational grade indicates the closeness between the ideal sequence and the comparability sequence. An alternative with the highest grey relational grade is the best choice.

\section{VlseKriterijumska Optimizacija I Kompromisno Resenje (VIKOR)}

VIKOR was proposed by Opricovic ${ }^{28}$ and Opricovic and Tzeng ${ }^{30}$ for multicriteria optimization of complex systems. The multicriteria ranking index, which is based on the particular measure of closeness to the ideal alternative, is introduced to rank alternatives in the presence of conflicting criteria. ${ }^{28,49}$ This paper uses the following VIKOR algorithm provided by Opricovic and Tzeng $^{29}$ in the experiment:

Step 1. Determine the best $f_{i}^{*}$ and the worst $f_{i}^{-}$values of all criterion functions, $i=1,2, \ldots, n$.

$$
\begin{aligned}
& f_{i}^{*}=\left\{\begin{array}{l}
\max _{j} f_{i j}, \text { for benefit criteria } \\
\min _{j} f_{i j}, \text { for cost criteria }
\end{array}\right\}, \quad j=1,2, \ldots, J, \\
& f_{i}^{-}=\left\{\begin{array}{l}
\min _{j} f_{i j}, \text { for benefit criteria } \\
\max _{j} f_{i j}, \text { for cost criteria }
\end{array}\right\}, \quad j=1,2, \ldots, J,
\end{aligned}
$$

where $J$ is the number of alternatives, $n$ is the number of criteria, and $f_{i j}$ is the rating of $i$ th criterion function for alternative $a_{j}$.

Step 2. Compute the values $S_{j}$ and $R_{j}, j=1,2, \ldots, J$, by the relations

$$
\begin{aligned}
S_{j} & =\sum_{i=1}^{n} w_{i}\left(f_{i}^{*}-f_{i j}\right) /\left(f_{i}^{*}-f_{i}^{-}\right), \\
R_{j} & =\max _{i}\left[w_{i}\left(f_{i}^{*}-f_{i j}\right) /\left(f_{i}^{*}-f_{i}^{-}\right)\right],
\end{aligned}
$$


where $w_{i}$ is the weight of $i$ th criteria, $S_{j}$ and $R_{j}$ are used to formulate ranking measure.

Step 3. Compute the values $Q_{j}, j=1,2, \ldots, J$, by the relations

$$
\begin{aligned}
Q_{j} & =v\left(S_{j}-S^{*}\right) /\left(S^{-}-S^{*}\right)+(1-v)\left(R_{j}-R^{*}\right) /\left(R^{-}-R^{*}\right), \\
S^{*} & =\min _{j} S_{j}, \quad S^{-}=\max _{j} S_{j}, \\
R^{*} & =\min _{j} R_{j}, \quad R^{-}=\max _{j} R_{j},
\end{aligned}
$$

where the solution obtained by $S^{*}$ is with a maximum group utility, the solution obtained by $R^{*}$ is with a minimum individual regret of the opponent, and $v$ is the weight of the strategy of the majority of criteria. The value of $v$ is set to 0.5 in the experiment.

Step 4. Rank the alternatives in decreasing order. There are three ranking lists: $S, R$, and $Q$.

Step 5. Propose the alternative $a^{\prime}$, which is ranked the best by $Q$, as a compromise solution if the following two conditions are satisfied:

(a) $Q\left(a^{\prime \prime}\right)-Q\left(a^{\prime}\right) \geq 1 /(J-1)$; (b) Alternative $a^{\prime}$ is ranked the best by $S$ or/ and $R$.

If only the condition (b) is not satisfied, alternatives $a^{\prime}$ and $a^{\prime \prime}$ are proposed as compromise solutions, where $a^{\prime \prime}$ is ranked the second by $Q$. If the condition (a) is not satisfied, alternatives $a^{\prime}, a^{\prime \prime}, \ldots, a^{M}$ are proposed as compromise solutions, where $a^{M}$ is ranked the Mth by $Q$ and is determined by the relation $Q\left(a^{M}\right)-Q\left(a^{\prime}\right)<$ $1 /(J-1)$ for maximum $\mathrm{M}$.

\section{Preference Ranking Organisation METHod for Enrichment of Evaluations (PROMETHEE)}

Brans $^{2}$ proposed the PROMETHEE I and PROMETHEE II in 1982. The PROMETHEE methods use pairwise comparisons and outranking relationships to choose the best alternatives. The final selection is based on the positive and negative preference flows of each alternative. The positive preference flow indicates how an alternative is outranking all the other alternatives and the negative preference flow indicates how an alternative is outranked by all the other alternatives. ${ }^{3}$ PROMETHEE I obtains partial ranking because it does not compare conflicting actions. ${ }^{4}$ PROMETHEE II ranks alternatives according to the net flow which equals to the balance of the positive and the negative preference flows. ${ }^{43}$ An alternative with a higher net flow is better. ${ }^{3}$ Since the purpose of this paper is to rank classification algorithms, PROMETHEE II is used in the experimental study. 
The following PROMETHEE II procedure described by Brans and Mareschal ${ }^{3}$ is used in the experimental study:

Step 1. Define aggregated preference indices.

Let $a, b \in A$, and let:

$$
\left\{\begin{array}{l}
\pi(a, b)=\sum_{j=1}^{k} p_{j}(a, b) w_{j} \\
\pi(b, a)=\sum_{j=1}^{k} p_{j}(b, a) w_{j}
\end{array}\right.
$$

where $A$ is a finite set of possible alternatives $\left\{a_{1}, a_{2}, \ldots, a_{n}\right\}, k$ represents the number of evaluation criteria and $w_{j}$ is the weight of each criterion. Arbitrary numbers for the weights can be assigned by the DM. The weights are then normalized to ensure that $\sum_{j=1}^{k} w_{j}=1 . \pi(a, b)$ indicates how $a$ is preferred to $b$ and $\pi(b, a)$ indicates how $b$ is preferred to $a . P_{j}(a, b)$ and $P_{j}(b, a)$ are the preference functions for alternatives $a$ and $b$.

Step 2. Calculate $\pi(a, b)$ and $\pi(b, a)$ for each pair of alternatives of $A$.

Step 3. Define the positive and the negative outranking flow as follows:

The positive outranking flow:

$$
\phi^{+}(a)=\frac{1}{n-1} \sum_{x \in A} \pi(a, x) .
$$

The positive outranking flow:

$$
\phi^{-}(a)=\frac{1}{n-1} \sum_{x \in A} \pi(x, a) .
$$

Step 4. Compute the net outranking flow for each alternative as follows:

$$
\phi(a)=\phi^{+}(a)-\phi^{-}(a) .
$$

When $\phi(a)>0, a$ is more outranking all the alternatives on all the evaluation criteria. When $\phi(a)<0, a$ is more outranked.

\subsection{Spearman's rank correlation coefficient}

Spearman's rank correlation coefficient measures the similarity between two sets of rankings. The basic idea of the proposed approach is to assign a weight to each MCDM method according to the similarities between the ranking it generated and the rankings produced by other MCDM methods. A large value of Spearman's rank correlation coefficient indicates a good agreement between a MCDM method and other MCDM methods.

The proposed approach is designed to handle conflicting MCDM rankings through three steps. In the first step, a selection of MCDM methods is applied 
to rank classification algorithms. If there are strong disagreements among MCDM methods, the different ranking scores generated by MCDM methods are used as inputs for the second step.

The second step utilizes Spearman's rank correlation coefficient to find the weights for each MCDM method. Spearman's rank correlation coefficient between the $k$ th and $i$ th MCDM methods is calculated by the following equation:

$$
\rho_{k i}=1-\frac{6 \sum d_{i}^{2}}{n\left(n^{2}-1\right)},
$$

where $n$ is the number of alternatives and $d_{i}$ is the difference between the ranks of two MCDM methods. Based on the value of $\rho_{k i}$, the average similarities between the $k$ th MCDM method and other MCDM methods can be calculated as

$$
\rho_{k}=\frac{1}{q-1} \sum_{i=1, i \neq k}^{q} \rho_{k i}, \quad k=1,2, \ldots, q,
$$

where $q$ is the number of MCDM methods. The larger the $\rho_{k}$ value, the more important the MCDM method is. Normalized $\rho_{k}$ values can then be used as weights for MCDM methods in the secondary ranking.

The third step uses the weights obtained from the second step to get secondary rankings of classifiers. Each MCDM method is applied to re-rank classification algorithms using ranking scores produced by MCDM methods in the first step and the weights obtained in the second step.

\section{Experimental Study}

The experiment chooses a wide selection of classification algorithms, datasets, and performance measures to test the proposed approach. The following subsections describe the data sources, classification algorithms, performance measures, and the experimental design.

\subsection{Data sources}

Eleven binary classification datasets are selected to evaluate the performance of classifiers. Table 1 displays the basic information of these datasets.

All datasets are publicly available at the UCI machine learning repository (http://archive.ics.uci.edu/ml/). These datasets are selected from a variety of disciplines, including life science, physical science, social science, and business, and represent a variation of data structures. Their sizes range from 208 to 19,020; the number of attributes varies from 4 to 73 ; and class distributions change from 93.6/6.4 to 52/48. By averaging the performances of classifiers across the 11 datasets, this study attempts to get a comprehensive evaluation of classification algorithms. 
Table 1. Summary of UCI datasets.

\begin{tabular}{lcccc}
\hline Dataset & No. of Instances & No. of Attributes & Area & Goods/Bads \\
\hline Adult & 48,842 & 14 & Social & $75.9 / 34.1$ \\
Breast-cancer (original) & 699 & 10 & Life & $65.5 / 34.5$ \\
Breast-cancer (diagnostic) & 569 & 32 & Life & $58 / 42$ \\
Glass & 214 & 10 & Physical & $52 / 48$ \\
Ionosphere & 351 & 34 & Physical & $64.1 / 35.9$ \\
Magic gamma telescope & 19,020 & 10 & Physical & $64.8 / 35.2$ \\
Mammographic & 961 & 5 & Life & $53.7 / 46.3$ \\
Ozone level detection & 2536 & 73 & Physical & $93.6 / 6.4$ \\
Pima Indians diabetes & 768 & 8 & Life & $65.1 / 34.9$ \\
Sonar & 208 & 60 & Physical & $53.4 / 46.6$ \\
Transfusion & 748 & 4 & Business & $76.2 / 23.8$ \\
\hline
\end{tabular}

\subsection{Classification algorithms}

Classification algorithms used in the experiment represent five categories: Trees, rules, Bayesian classifiers, lazy classifiers, and miscellaneous classifiers. All classifiers are implemented in WEKA. ${ }^{45}$

Trees category includes C4.5 decision tree, decision stump, random tree, and REP tree. C4.5 is a decision tree algorithm that constructs decision trees in a topdown recursive divide-and-conquer manner ${ }^{36}$ and is a commonly used benchmark classifier. An algorithm for generating grafted C4.5 decision trees is also included. ${ }^{46}$ Decision stump is a simple one-level decision tree and often used with ensemble techniques. ${ }^{18}$ Random tree builds trees by choosing a test based on number of random features at each node. ${ }^{45}$ REP tree uses information gain to build a decision tree and reduced-error pruning (REP) to prune it. ${ }^{45}$

Rules category includes the zero-attribute rule, conjunctive rule, decision table, one-attribute rule, and part. The zero-attribute rule, or ZeroR, predicts the mode and is often used to measure the performance of other algorithms. The conjunctive rule learns a single best rule to predict a class value and assign uncovered test instances to the default class value of the uncovered training instances. ${ }^{45}$ Decision table builds a decision table majority classifier by selecting the right feature subsets. Instances not covered by a decision table can be determined by the nearest-neighbor method. The one-attribute rule, or OneR, finds association rules using just one attribute. ${ }^{17}$ Part generates partial C4.5 decision trees and obtains rules from the partial trees. ${ }^{10}$

Bayesian classifiers category includes Bayesian network ${ }^{47}$ and Naïve Bayes. ${ }^{6}$ Both classifiers model probabilistic relationships between predictor variables and the class variable. While Naïve Bayes classifier estimates the class-conditional probability based on Bayes theorem and can only represent simple distributions, Bayesian network is a probabilistic graphic model and can represent conditional independencies between variables. Two forms of Naïve Bayes classifier are included in this study: the standard probabilistic Naïve Bayes classifier and an incremental Naïve Bayes classifier. ${ }^{45}$ 
IB1, a basic instance-based learner provided by WEKA, is chosen to represent lazy classifiers. An unknown instance is assigned to the same class as the training instance that is the closest to it measured by Euclidean distance.

In addition to the above mentioned classifiers, Adaboost M1, hyperpipe, and voting feature interval (VFI) are included. Adaboost M1 algorithm changes weights of training instances in each iteration to force learning algorithms to put more emphasis on previously misclassified instances. ${ }^{12}$ Hyperpipe classifier constructs a hyperpipe for each category and assigns a new instance to the category that most contains the instance. ${ }^{45}$ VFI classifier creates a set of feature intervals and records class counts for each interval. The class having the highest vote is the predicted class. $^{13}$

\subsection{Performance measures}

Widely used performance measures in classification are accuracy, true positive rate, true negative rate, mean absolute error (MAE), precision, F-measure, the area under receiver operating characteristic (AUC), kappa statistic, and computation time. Definitions of these measures are provided as follows.

- Overall accuracy: Accuracy is the percentage of correctly classified instances.

$$
\text { Overall accuracy }=\frac{\mathrm{TN}+\mathrm{TP}}{\mathrm{TP}+\mathrm{FP}+\mathrm{FN}+\mathrm{TN}},
$$

where TN, TP, FN, and FP stand for true negative, true positive, false negative, and false positive, respectively.

- True Positive (TP): TP is the number of correctly classified positive instances. $\mathrm{TP}$ rate is also called sensitivity measure.

$$
\text { True Positive rate/Sensitivity }=\frac{\mathrm{TP}}{\mathrm{TP}+\mathrm{FN}} .
$$

- False Positive (FP): FP is the number of positive instances that is misclassified as negative class. FP rate is called Type-I error.

$$
\text { False Positive rate/Type-I error }=\frac{\mathrm{FP}}{\mathrm{FP}+\mathrm{TN}} \text {. }
$$

- True Negative (TN): TN is the number of correctly classified negative instances. $\mathrm{TN}$ rate is also called specificity measure.

$$
\text { True Negative rate/Specificity }=\frac{\mathrm{TN}}{\mathrm{TN}+\mathrm{FP}} .
$$

- False Negative (FN): FN is the number of negative instances that is misclassified as positive class. FN rate is also called Type-II error.

$$
\text { False Negative rate/Type-II error }=\frac{\mathrm{FN}}{\mathrm{FN}+\mathrm{TP}} .
$$


- Mean absolute error (MAE): This measures how much the predictions deviate from the true probability. $P(i, j)$ is the estimated probability of $i$ module to be of class $j$ taking values in $[0,1] .^{9}$

$$
\mathrm{MAE}=\frac{\sum_{j=1}^{c} \sum_{i=1}^{m}|f(i, j)-P(i, j)|}{m \cdot c} .
$$

- Precision: This is the number of classified fault-prone modules that actually are fault-prone modules.

$$
\text { Precision }=\frac{\mathrm{TP}}{\mathrm{TP}+\mathrm{FP}}
$$

- F-measure: It is the harmonic mean of precision and recall. F-measure has been widely used in information retrieval. ${ }^{1}$

$$
\text { F-measure }=\frac{2 \times \text { Precision } \times \text { Recall }}{\text { Precision }+ \text { Recall }} .
$$

- AUC: ROC stands for receiver operating characteristic, which shows the tradeoff between TP rate and FP rate. AUC represents the accuracy of a classifier. The larger the area, the better the classifier.

- Kappa statistic (KapS): This is a classifier performance measure that estimates the similarity between the members of an ensemble in multi-classifiers systems. ${ }^{21}$

$$
\mathrm{KapS}=\frac{P(A)-P(E)}{1-P(E)} .
$$

$\mathrm{P}(\mathrm{A})$ is the accuracy of the classifier and $\mathrm{P}(\mathrm{E})$ is the probability that agreement among classifiers is due to chance.

$$
\mathrm{P}(\mathrm{E})=\frac{\sum_{k=1}^{c}\left(\left[\sum_{j=1}^{c} \sum_{i=1}^{m} f(i, k) C(i, j)\right] \cdot\left[\sum_{j=1}^{c} \sum_{i=1}^{m} f(i, j) C(i, k)\right]\right)}{m^{2}},
$$

where $m$ is the number of modules and $c$ is the number of classes. $f(i, j)$ is the actual probability of $i$ module to be of class $j$. $\sum_{i=1}^{m} f(i, j)$ is the number of modules of class $j$. Given threshold $\theta, C_{\theta}(i, j)$ is 1 if $j$ is the predicted class for $i$ obtained from $P(i, j)$; otherwise it is $0 .^{9}$

- Training time: The time needed to train a classification algorithm or ensemble method.

- Test time: The time needed to test a classification algorithm or ensemble method.

\subsection{Experimental design}

The experiment was carried out according to the following process:

Input: 11 binary classification datasets

Output: Rankings of classifiers 
Step 1. Prepare target datasets: Select and transform relevant features; data cleaning; data integration.

Step 2. Train and test the selected classification algorithms on a randomly sampled partitions (i.e., 10-fold cross-validation) using WEKA 3.7. ${ }^{16}$

Step 3. Evaluate classification algorithms using TOPSIS, GRA, VIKOR, PROMETHEE II, and ELECTRE III. MCDM methods are implemented using MATLAB $7.0 .^{25}$

Step 4. Generate the first ranking of classification algorithms provided by each MCDM method. If there are disagreements among MCDM methods, go to Step 5; otherwise, end the process.

Step 5. Solve the formulas (2.4) and (2.5) to get the weights of MCDM methods.

Step 6. Recalculate the rankings of classifiers using the MCDM methods with the ranking produced in Step 4 and the normalized weights obtained in Step 5 as inputs.

\section{END}

Classification algorithm selection problem involves both benefit and cost criteria. A criterion is called the benefit because the higher a classification algorithm scores in terms of the corresponding criterion, the better the algorithm is. Seven of the 10 performance metrics used in this study are benefit criteria. They are accuracy, kappa statistic, TP rate, TN rate, precision, F-measure, and AUC. On the other hand, a criterion is called the cost because the higher a classification algorithm scores in terms of the corresponding criterion, the worse the algorithm is. ${ }^{44}$ Three performance metrics used in this study are cost criteria: MAE, training, and testing time. For TOPISIS, ELECTRE III, and PROMETHEE II, criteria or performance measures are assigned equal weights.

The proposed approach is tested on two individual UCI datasets and all 11 datasets. Classification algorithms are first applied to two UCI datasets (i.e., adult and magic gamma telescope) and evaluated using the proposed ranking approach. Then the performances of these classifiers on the 11 UCI datasets are averaged and ranked using the proposed ranking approach. The classification results and the first and secondary rankings of classifiers are reported in the following section.

\section{Results}

\subsection{Classification results}

\subsubsection{Magic gamma telescope dataset}

Table 2 shows the testing results of the magic gamma telescope dataset using the selected classifiers. Each classifier is measured by the ten performance metrics defined in Sec. 3.3. The best result of each performance measure is highlighted in boldface. Adaboost M1 algorithm performances well on this dataset and there is no classification algorithm achieves the best results across all measures. 


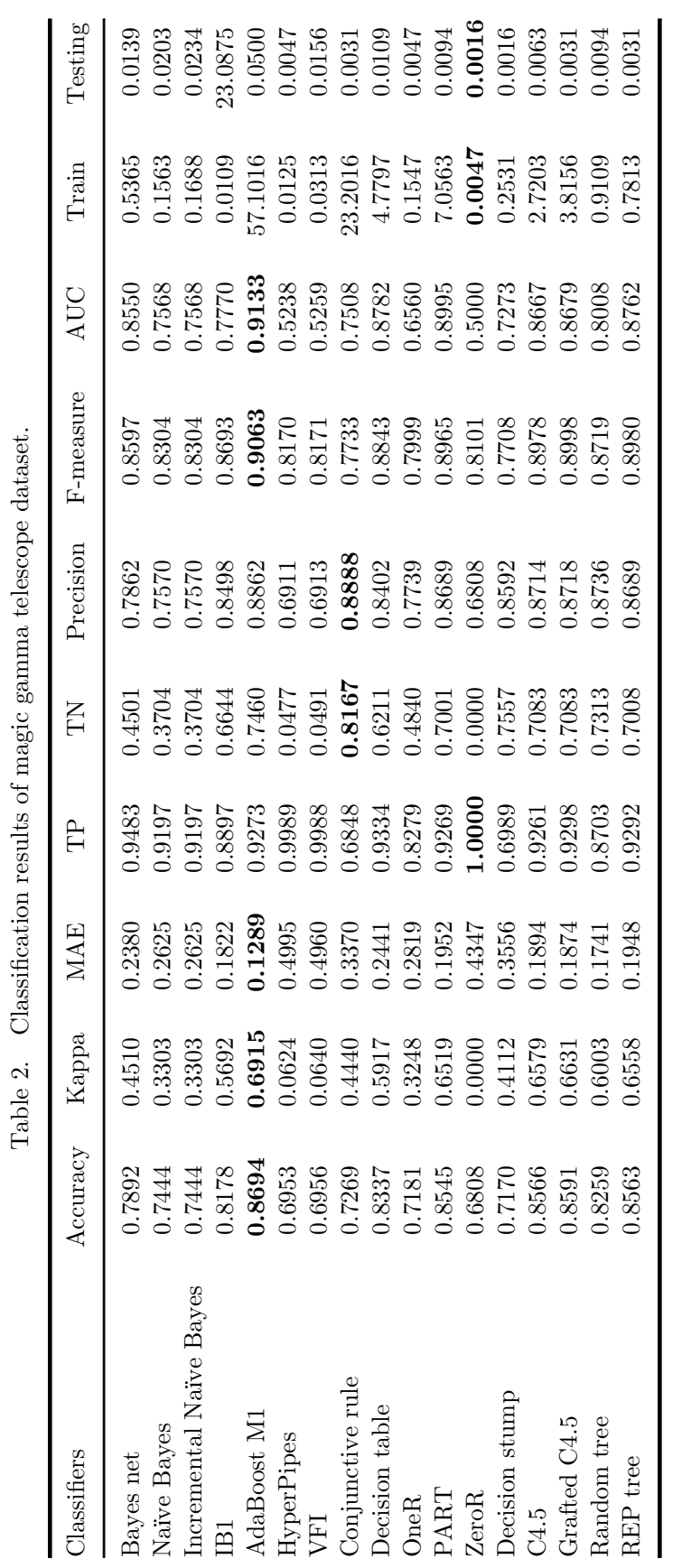




\subsubsection{Adult dataset}

Table 3 summarizes the testing results of the adult dataset using the classifiers. The best result of each performance measure is highlighted in boldface. Similar to the magic gamma telescope data, there is no classification algorithm that has the best results on all measures, and the performances of classifiers on this dataset are more divergent than on the magic gamma telescope data. Adaboost M1 algorithm, which performances significantly better than other classifiers on the magic gamma telescope data, only achieves the best MAE on the adult dataset.

\subsubsection{Average of 11 datasets}

Table 4 summarizes the testing results of the adult dataset using the selected 17 classifiers. The best result of each performance measure is highlighted in boldface. Similar to the previous two datasets, best performance measures are achieved by difference classification algorithms.

\subsection{MCDM rankings}

Following the proposed approach and the experimental design, the five MCDM methods are applied to rank 17 classification algorithms using the classification results of the magic gamma telescope, adult, and the average of all 11 datasets.

\subsubsection{Magic gamma telescope dataset}

The ranking scores of classifiers generated by TOPSIS, GRA, VIKOR, PROMETHEE II, and ELECTRE III for the magic gamma telescope dataset are summarized in Table 5. Each MCDM method provides a value and ranking for each classifier based on their performances on the testing data.

For the magic gamma telescope data, five MCDM methods all rank Adaboost M1 algorithm as the best classifier. However, their rankings for IB1 are quite different. While TOPSIS ranks IB1 as the second best classifier for this datasets, VIKOR, PROMETHEE, and ELECTRE rank it as the 8th classifier. In fact, the five MCDM methods agree on only three classifiers: Adaboost M1, HyperPipes, and ZeroR.

\subsubsection{Adult data}

The ranking scores of classifiers generated by TOPSIS, GRA, VIKOR, PROMETHEE II, and ELECTRE III for the adult dataset are summarized in Table 6. The five MCDM methods agree on no classifier over the adult dataset. The ranking results can be expected since the performances of the classifiers on the adult data are divergent (see Table 3).

\subsubsection{Average of 11 datasets}

Table 7 reports the rankings of classifiers generated by the five MCDM methods based on their average classification results on 11 datasets. Similar to the adult dataset, the five MCDM methods cannot reach an agreement on any classifier. 


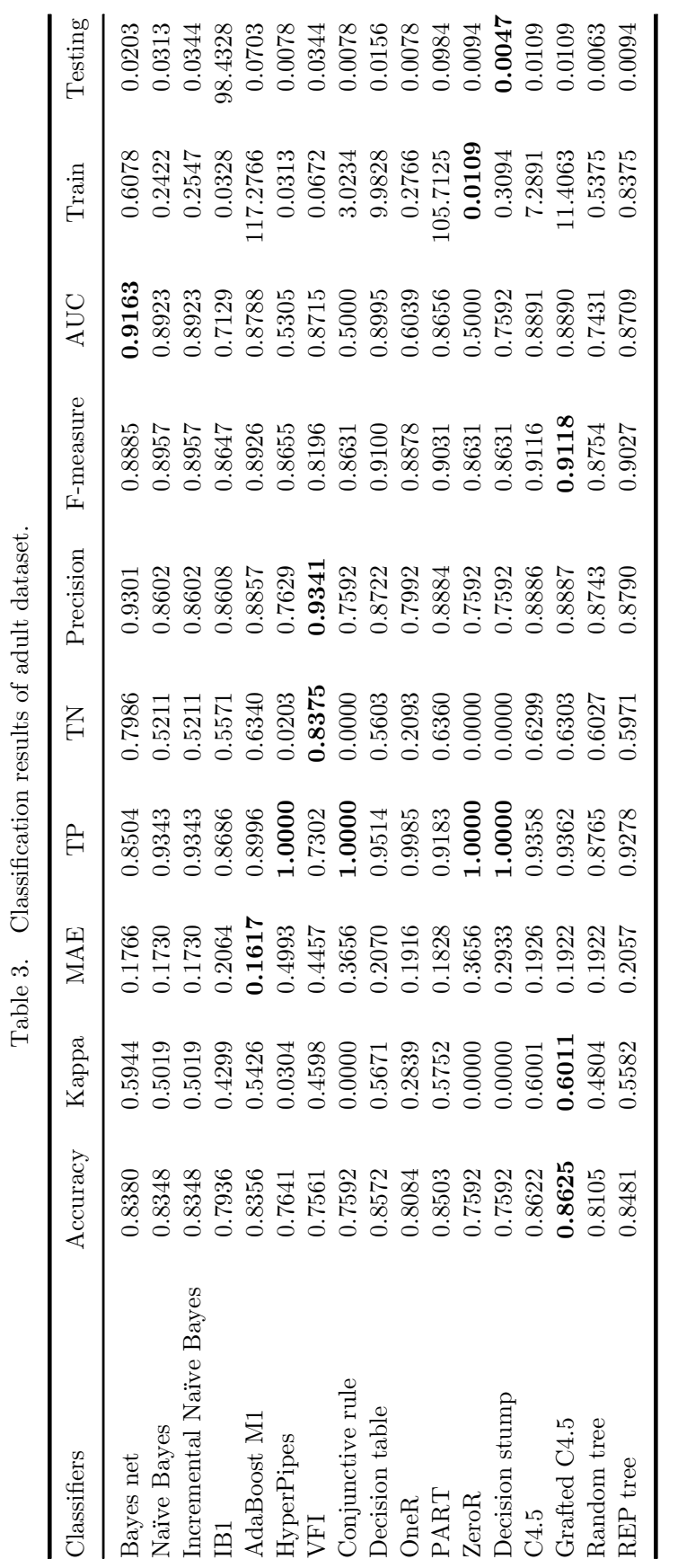




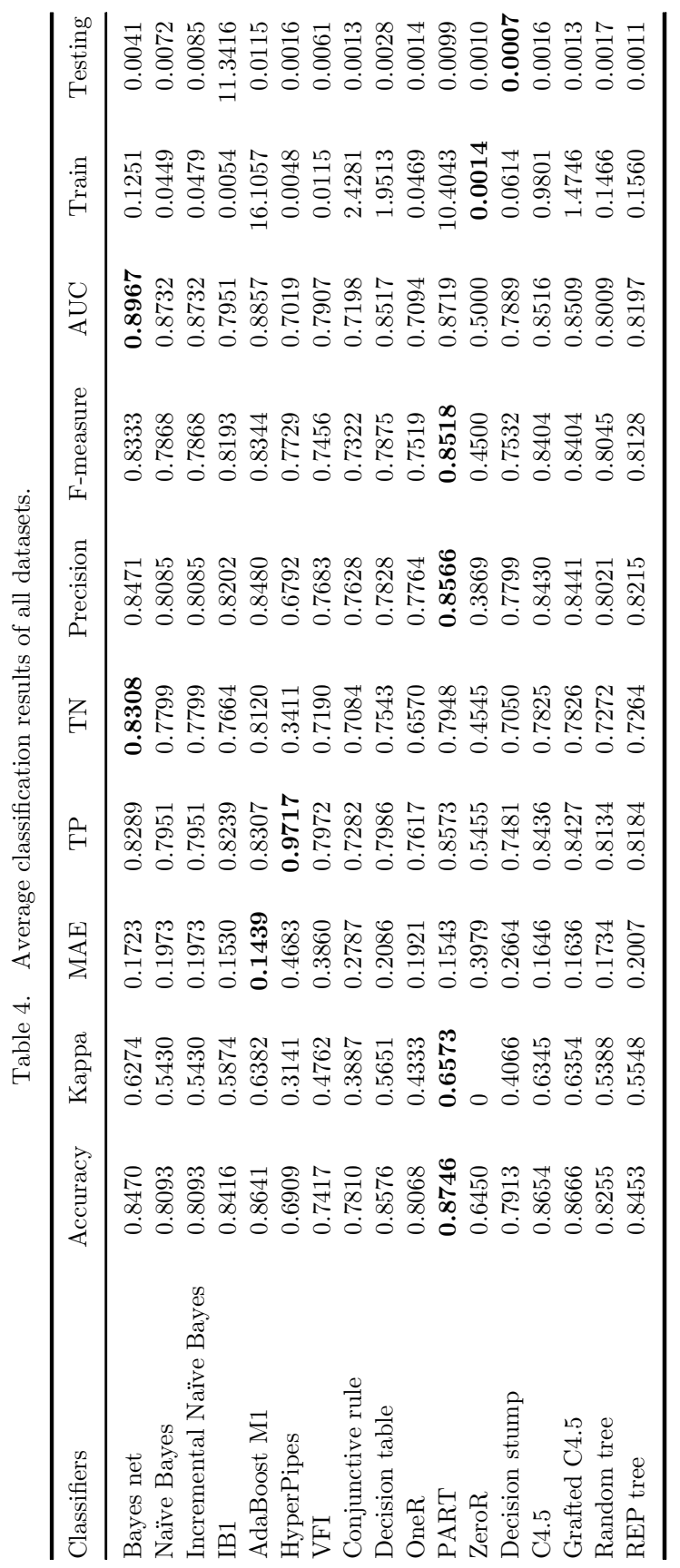




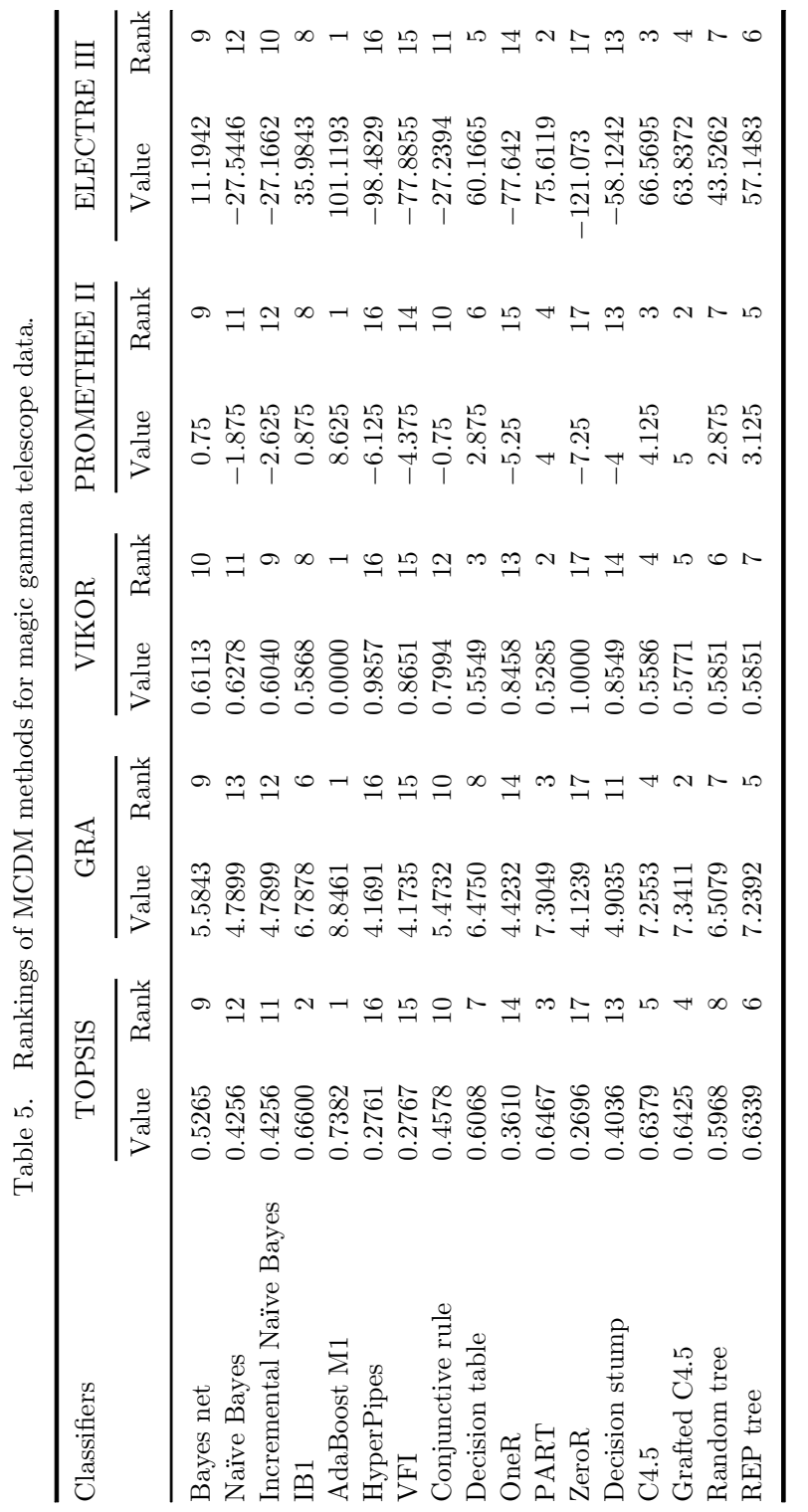




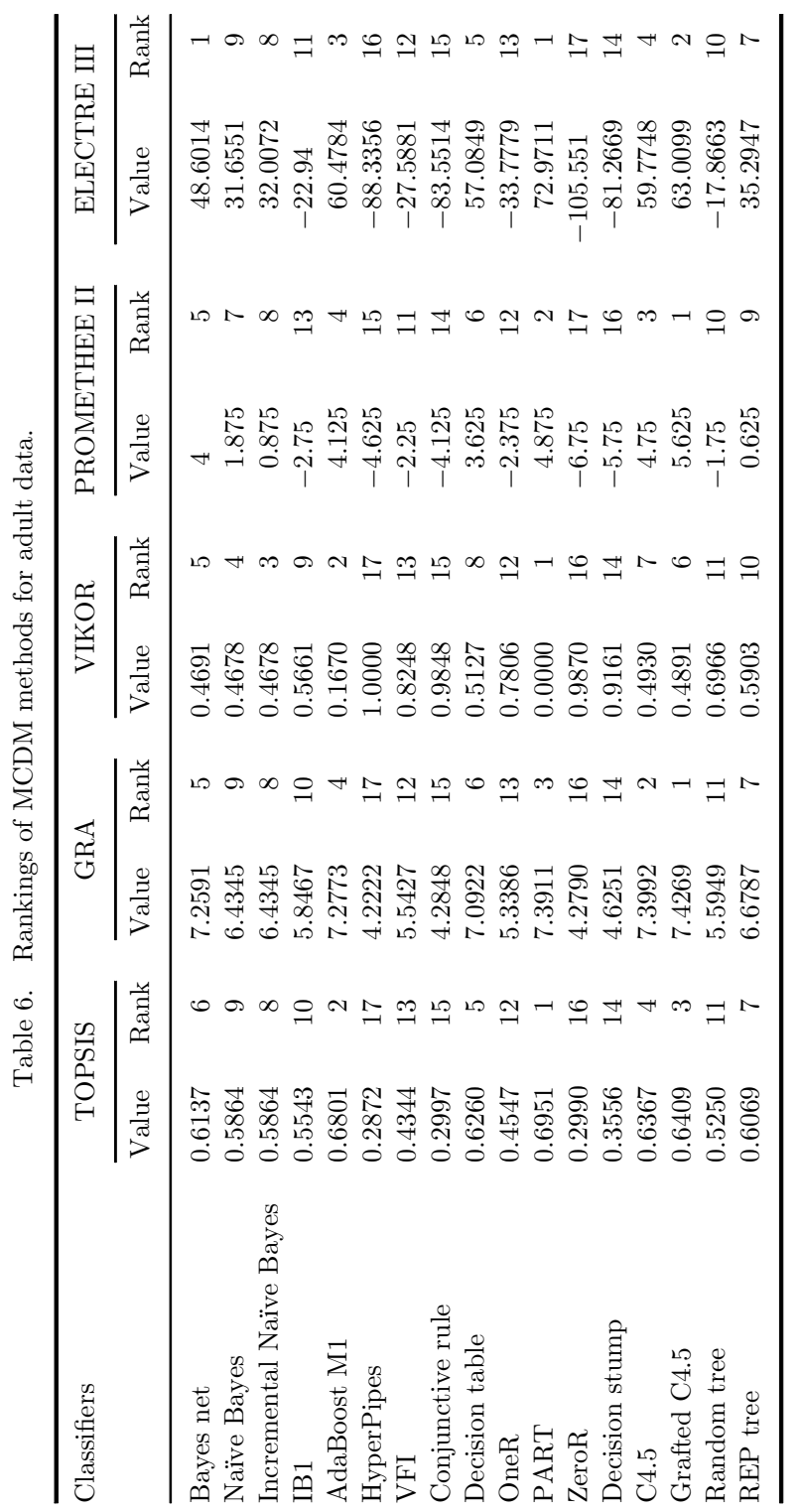




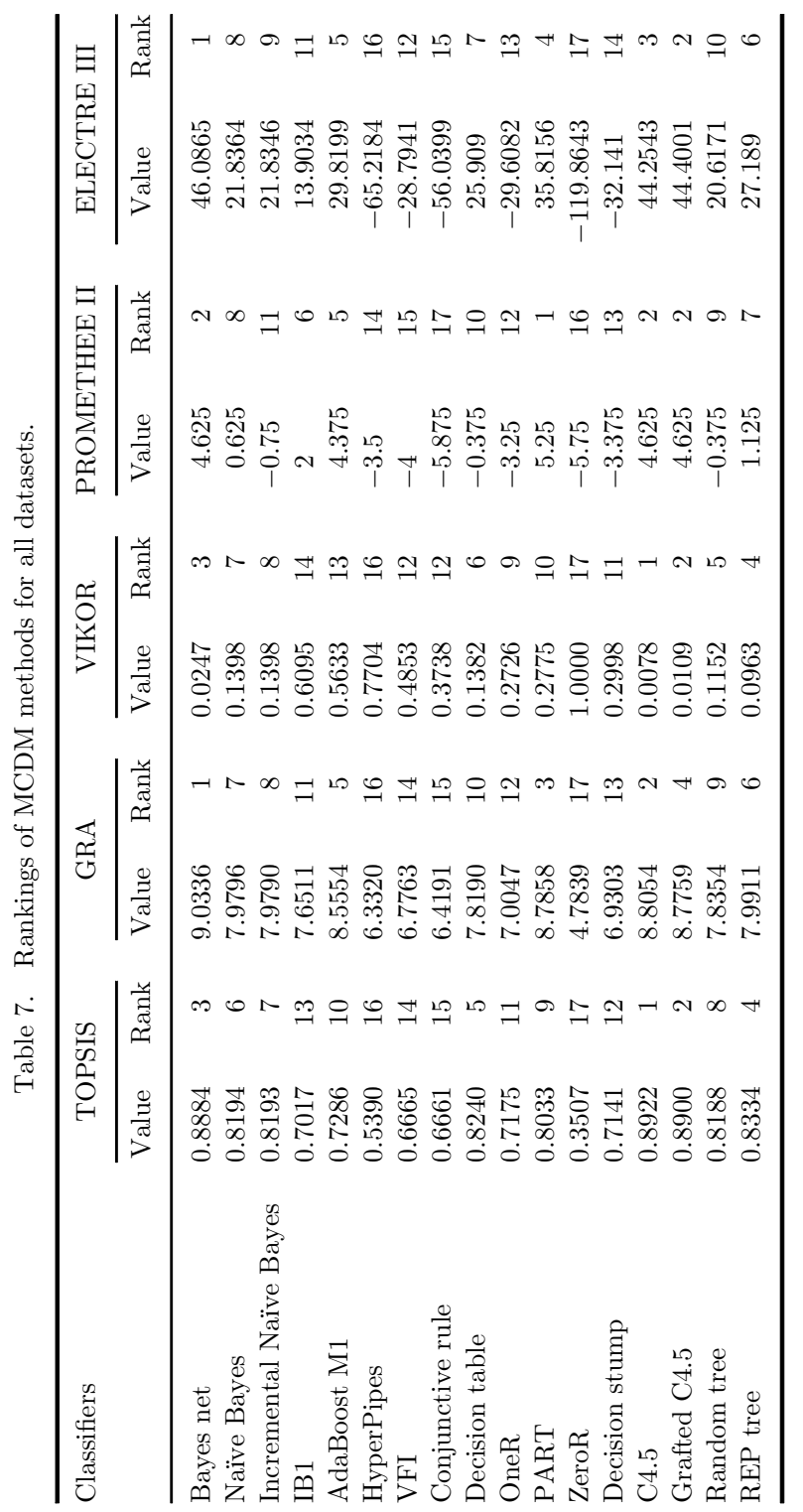


Results of the three tests indicate that different MCDM methods may have strong disagreements. Therefore, the next step applies Spearman's rank correlation coefficient to generate secondary rankings in an attempt to resolve the inconsistency.

\subsection{Secondary MCDM rankings}

The goal of the secondary ranking is to determine a set of weights for MCDM methods by solving the formulas (2.4) and (2.5). Before the computation, the ranking scores of the MCDM methods need to be standardized. The ranking scores of TOPSIS, GRA, PROMETHEE II, and ELECTRE III are standardized using (x-min)/(max-min); and the $\mathrm{Q}$ ranking scores of VIKOR are standardized using (max-x)/(max-min). The standardized MCDM ranking scores for the magic gamma telescope, adult, and the 11 datasets are presented in Tables 8-10, respectively.

\subsubsection{Magic gamma telescope dataset}

The standardized ranking scores are used to calculate the weights and normalized weights of the MCDM methods. The results are presented in Table 11.

Based on the standardized ranking scores and the normalized weights of the MCDM methods, the secondary rankings of classifiers on the magic gamma telescope dataset are computed and summarized in Table 12. The five MCDM methods provide the same rankings for 11 classifiers. Rankings of TOPSIS and GRA agree on all the classifiers, and differ from VIKOR and PROMETHEE II on only two classifiers. The degrees of disagreements on the rankings of classifiers among MCDM methods are also reduced.

Table 8. Standardized MCDM ranking scores for magic gamma telescope dataset.

\begin{tabular}{llllcc}
\hline Classifiers & TOPSIS & GRA & VIKOR & PROMETHEE II & ELECTRE III \\
\hline Bayes net & 0.5482 & 0.3093 & 0.3887 & 0.5039 & 0.5953 \\
Naïve Bayes & 0.3328 & 0.141 & 0.3722 & 0.3386 & 0.4209 \\
Incremental Naïve Bayes & 0.3329 & 0.141 & 0.396 & 0.2913 & 0.4226 \\
IB1 & 0.833 & 0.5641 & 0.4132 & 0.5118 & 0.7069 \\
AdaBoost M1 & 1 & 1 & 1 & 1 & 1 \\
HyperPipes & 0.0138 & 0.0096 & 0.0143 & 0.0709 & 0.1017 \\
VFI & 0.0153 & 0.0105 & 0.1349 & 0.1811 & 0.1944 \\
Conjunctive rule & 0.4018 & 0.2857 & 0.2006 & 0.4094 & 0.4223 \\
Decision table & 0.7197 & 0.4979 & 0.4451 & 0.6378 & 0.8157 \\
OneR & 0.195 & 0.0634 & 0.1542 & 0.126 & 0.1955 \\
PART & 0.8048 & 0.6736 & 0.4715 & 0.7087 & 0.8852 \\
ZeroR & 0 & 0 & 0 & 0 & 0 \\
Decision stump & 0.2859 & 0.1651 & 0.1451 & 0.2047 & 0.2833 \\
C4.5 & 0.7859 & 0.6631 & 0.4414 & 0.7165 & 0.8445 \\
Grafted C4.5 & 0.7958 & 0.6813 & 0.4229 & 0.7717 & 0.8322 \\
Random tree & 0.6983 & 0.5049 & 0.4149 & 0.6378 & 0.7408 \\
REP tree & 0.7775 & 0.6597 & 0.4149 & 0.6535 & 0.8021 \\
\hline
\end{tabular}


Table 9. Standardized MCDM ranking scores for adult dataset.

\begin{tabular}{llllcc}
\hline Classifiers & TOPSIS & GRA & VIKOR & PROMETHEE II & ELECTRE III \\
\hline Bayes net & 0.8005 & 0.9476 & 0.5309 & 0.8687 & 0.8635 \\
Naïve Bayes & 0.7336 & 0.6903 & 0.5322 & 0.697 & 0.7686 \\
Incremental Naïve Bayes & 0.7336 & 0.6903 & 0.5322 & 0.6162 & 0.7705 \\
IB1 & 0.6549 & 0.5069 & 0.4339 & 0.3232 & 0.4628 \\
AdaBoost M1 & 0.9633 & 0.9533 & 0.833 & 0.8788 & 0.93 \\
HyperPipes & 0 & 0 & 0 & 0.1717 & 0.0964 \\
VFI & 0.361 & 0.412 & 0.1752 & 0.3636 & 0.4367 \\
Conjunctive rule & 0.0308 & 0.0195 & 0.0152 & 0.2121 & 0.1232 \\
Decision table & 0.8307 & 0.8956 & 0.4873 & 0.8384 & 0.911 \\
OneR & 0.4106 & 0.3484 & 0.2194 & 0.3535 & 0.402 \\
PART & 1 & 0.9888 & 1 & 0.9394 & 1 \\
ZeroR & 0.0289 & 0.0177 & 0.013 & 0 & 0 \\
Decision stump & 0.1678 & 0.1257 & 0.0839 & 0.0808 & 0.136 \\
C4.5 & 0.8569 & 0.9914 & 0.507 & 0.9293 & 0.9261 \\
Grafted C4.5 & 0.8672 & 1 & 0.5109 & 1 & 0.9442 \\
Random tree & 0.583 & 0.4283 & 0.3034 & 0.404 & 0.4912 \\
REP tree & 0.7838 & 0.7665 & 0.4097 & 0.596 & 0.789 \\
\hline
\end{tabular}

Table 10. Standardized MCDM ranking scores for all datasets.

\begin{tabular}{llllcc}
\hline Classifiers & TOPSIS & GRA & VIKOR & PROMETHEE II & ELECTRE III \\
\hline Bayes net & 0.9929 & 1 & 0.983 & 0.9438 & 1 \\
Naïve Bayes & 0.8654 & 0.752 & 0.8669 & 0.5843 & 0.8539 \\
Incremental Naïve Bayes & 0.8654 & 0.7518 & 0.8669 & 0.4607 & 0.8539 \\
IB1 & 0.6481 & 0.6747 & 0.3936 & 0.7079 & 0.8061 \\
AdaBoost M1 & 0.6978 & 0.8875 & 0.4401 & 0.9213 & 0.902 \\
HyperPipes & 0.3478 & 0.3643 & 0.2314 & 0.2135 & 0.3293 \\
VFI & 0.5831 & 0.4688 & 0.5187 & 0.1685 & 0.5488 \\
Conjunctive rule & 0.5824 & 0.3848 & 0.6311 & 0 & 0.3846 \\
Decision table & 0.874 & 0.7142 & 0.8685 & 0.4944 & 0.8784 \\
OneR & 0.6773 & 0.5226 & 0.7331 & 0.236 & 0.5439 \\
PART & 0.8358 & 0.9417 & 0.7282 & 1 & 0.9381 \\
ZeroR & 0 & 0 & 0 & 0.0112 & 0 \\
Decision stump & 0.671 & 0.5051 & 0.7056 & 0.2247 & 0.5286 \\
C4.5 & 1 & 0.9463 & 1 & 0.9438 & 0.989 \\
Grafted C4.5 & 0.9959 & 0.9394 & 0.9968 & 0.9438 & 0.9898 \\
Random tree & 0.8644 & 0.7181 & 0.8917 & 0.4944 & 0.8465 \\
REP tree & 0.8914 & 0.7547 & 0.9108 & 0.6292 & 0.8861 \\
\hline
\end{tabular}

Table 11. Weights of MCDM methods for magic gamma telescope dataset.

\begin{tabular}{llcccc}
\hline & TOPSIS & GRA & VIKOR & PROMETHEE II & ELECTRE III \\
\hline Weights & 0.93811 & 0.95343 & 0.93689 & 0.95772 & 0.9663 \\
$\begin{array}{c}\text { Normalized } \\
\text { weights }\end{array}$ & 0.1974 & 0.20062 & 0.19714 & 0.20152 & 0.20333 \\
\hline
\end{tabular}



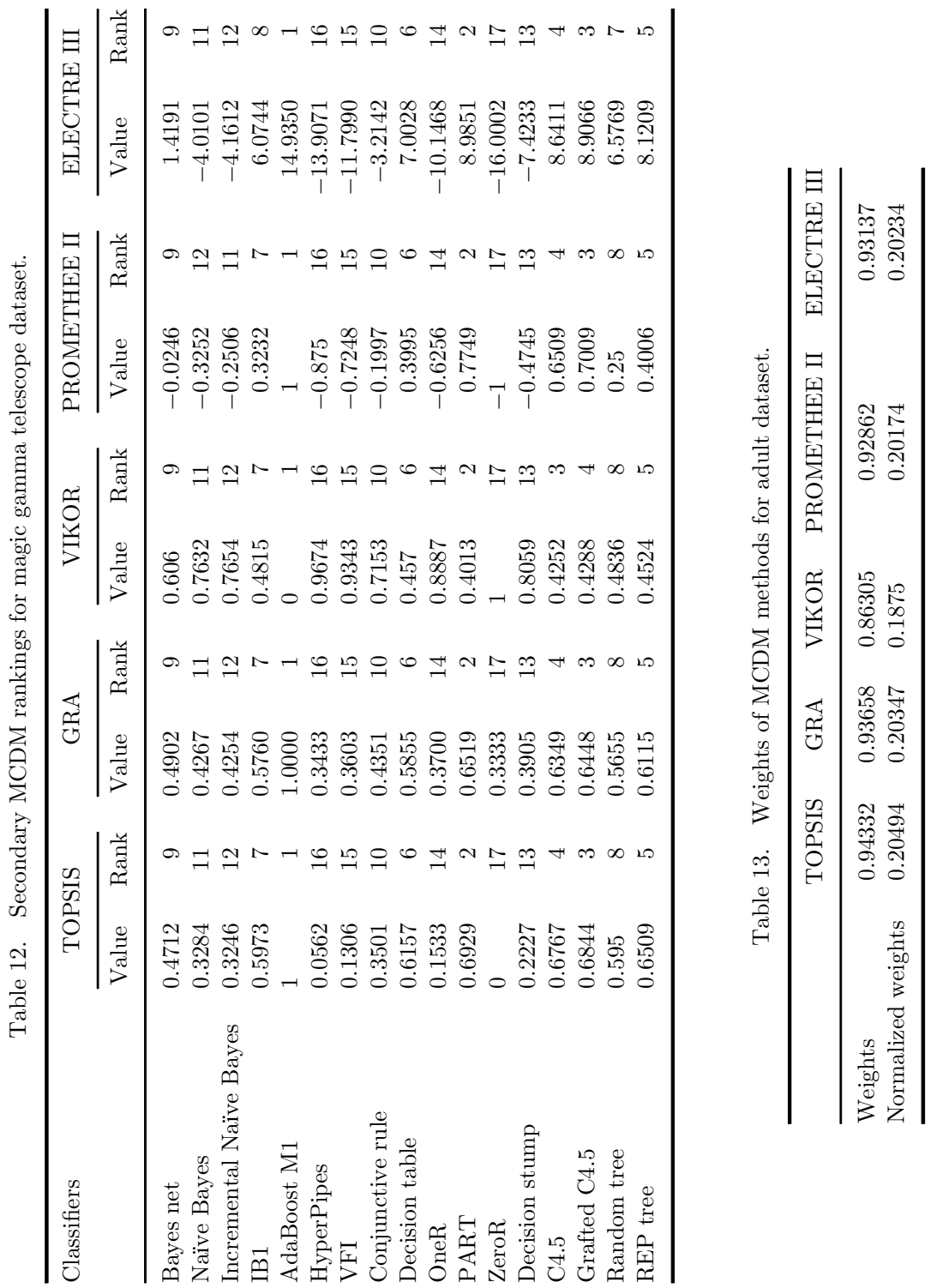


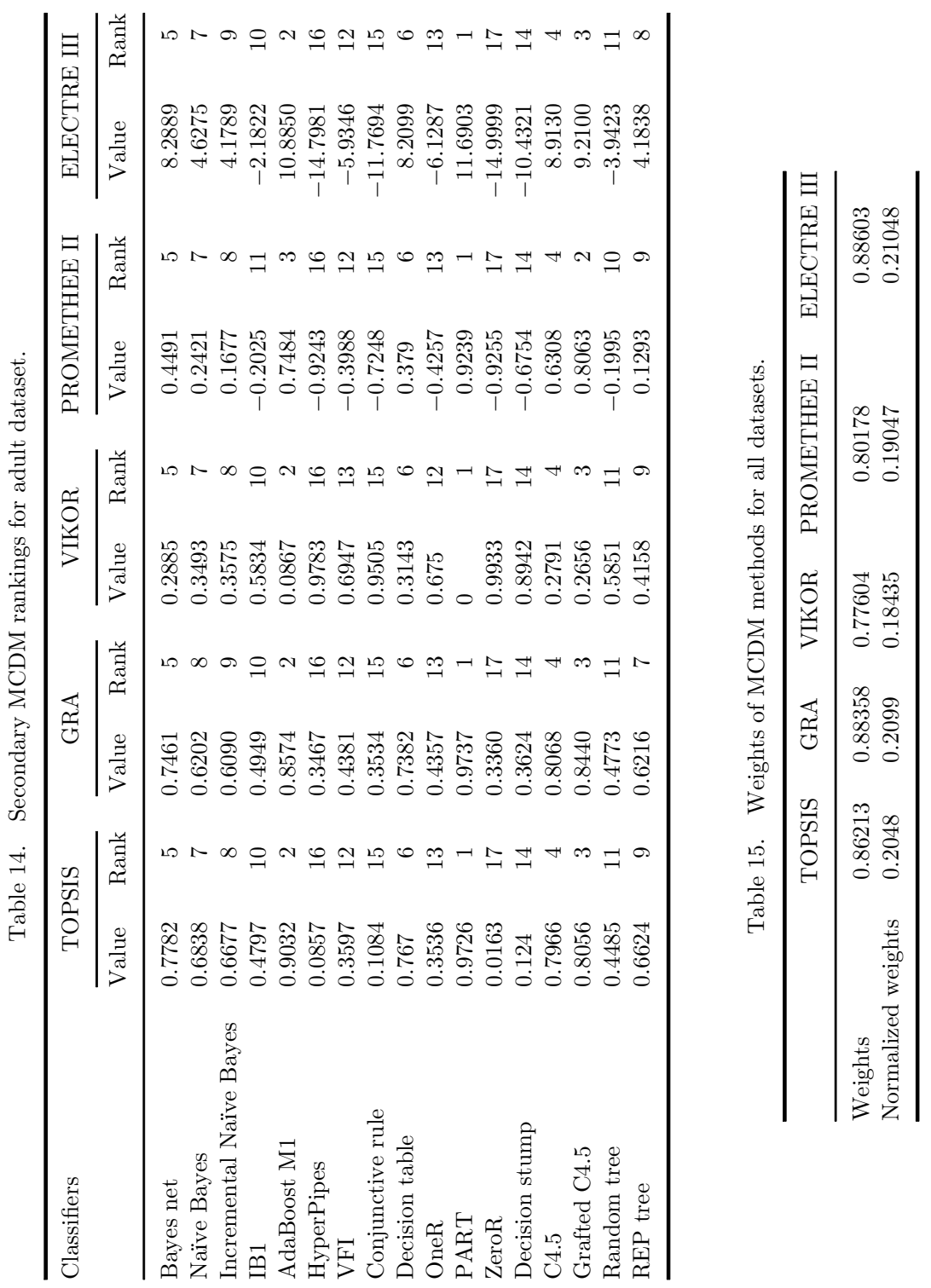




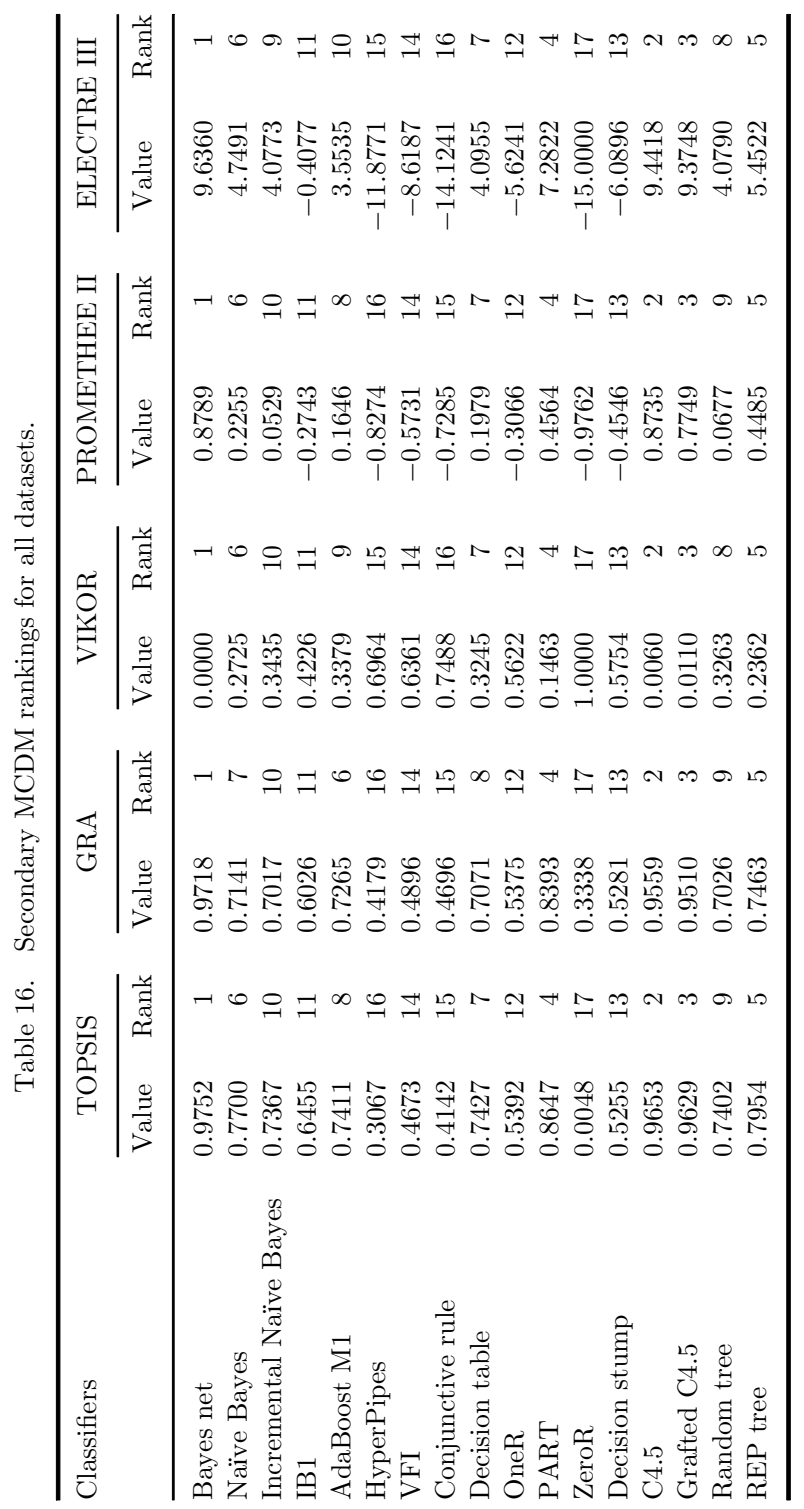




\subsubsection{Adult dataset}

Table 13 summarizes the weights and normalized weights of the five MCDM methods for the adult data.

The secondary rankings of classifiers on adult dataset are reported in Table 14. Ten classifiers got the same rankings on this dataset. The degrees of disagreements on the rankings of classifiers are greatly reduced.

\subsubsection{Average of 11 datasets}

The weights and normalized weights of the five MCDM methods for all 11 datasets are shown in Table 15.

The secondary rankings of classifiers on all the dataset are reported in Table 16. The five MCDM methods generate the same rankings on 10 classifiers. Rankings of classifiers produced by TOPSIS and PROMETHEE II are the same. The degrees of disagreements on the rankings of classifiers are greatly reduced compared with the original MCDM rankings.

The results of magic gamma telescope, adult, and all 11 datasets indicate that the secondary rankings of the MCDM methods are now in strong agreement. Specifically, classifiers that got the same rankings have increased from three to 11 for the magic gamma telescope dataset. For adult and the average of 11 datasets, the agreements of the five MCDM methods on classifiers have changed from zero to 10. Though the rankings of classifiers are not identical, the differences among the five MCDM methods have been largely reduced.

\section{Conclusion}

MCDM methods are feasible tools for selecting classification algorithms because the task of algorithms selection normally involves more than one criterion and can be modeled as MCDM problems. Since different MCDM methods evaluate classifiers from different aspects, they may produce divergent rankings.

This paper proposed an approach that uses Spearman's rank correlation coefficient to reconcile conflicting rankings generated by different MCDM methods for classification algorithms. The key of the proposed approach is to determine a weight for each MCDM method according to the similarities between the ranking it generated and the rankings produced by other MCDM methods. An MCDM method that has a larger Spearman's rank correlation coefficient is considered more important than one with a smaller Spearman's rank correlation coefficient because it has better agreements with other MCDM methods.

An experimental study was designed to validate the proposed approach. First, 17 classifiers were applied to 11 binary UCI classification datasets and the classification results were measured using 10 performance measures. Second, five MCDM methods (i.e., TOPSIS, GRA, VIKOR, PROMETHEE II, and ELECTRE III) were used to evaluate the classification algorithms based on their performances on the 
measures over magic gamma telescope data, adult data, and the collection of all 11 datasets. The rankings produced by the five MCDM methods for the three data are quite different. Third, the weights of MCDM methods were calculated using Spearman's rank correlation coefficient equations. The normalized weights and the rankings generated at the second stage were used as inputs to produce the secondary rankings of classifiers. The results indicate that the approach proposed can provide a compatible ranking when different MCDM techniques disagree.

\section{Acknowledgments}

This research has been partially supported by grants from the National Natural Science Foundation of China (\#70901015 for Gang Kou, \#70901011 and \#71173028 for Yi Peng, and \#70921061 for Yong Shi).

\section{References}

1. R. Baeza-Yates and B. Ribeiro-Neto, Modern Information Retrieval (Addison Wesley, 1999).

2. J. P. Brans, L'ingénièrie de la décision, Elaboration d'instruments d'aide à la décision. La méthode PROMETHEE, in L'aide à la décision: Nature, Instruments et Perspectives d'Avenir, eds. R. Nadeau and M. Landry (Presses de l'Université Laval, Québec, Canada, 1982), pp. 183-213.

3. J. P. Brans and B. Mareschal, PROMETHEE methods, in Multiple Criteria Decision Analysis: State of the Art Surveys, eds. J. Figueira, V. Mousseau and B. Roy (Springer, New York, 2005), pp. 163-195.

4. J. P. Brans and B. Mareschal, How to decide with PROMETHEE (1994), http://www. visualdecision.com/Pdf/How\%20to\%20use\%20PROMETHEE.pdf.

5. J. Deng, Control problems of grey systems, Systems and Control Letters 1 (1982) $288-294$.

6. P. Domingos and M. Pazzani, On the optimality of the simple Bayesian classifier under zero-one loss, Machine Learning 29(203) (1997) 103-130.

7. D. Ergu, G. Kou, Y. Peng and Y. Shi, A simple method to improve the consistency ratio of the pair-wise comparison matrix in ANP, European Journal of Operational Research 213(1) (2011) 246-259, doi:10.1016/j.ejor.2011.03.014.

8. D. Ergu, G. Kou, Y. Shi and Y. Shi, Analytic network process in risk assessment and decision analysis, Computers \& Operations Research (2011), doi:10.1016/j. cor.2011.03.005.

9. C. Ferri, J. Hernandezorallo and R. Modroiu, An experimental comparison of performance measures for classification, Pattern Recognition Letters 30(1) (2009) 27-38.

10. E. Frank and I. H. Witten, Generating accurate rule sets without global optimization, Fifteenth Int. Conf. Machine Learning (1998), pp. 144-151.

11. J. Figueira, V. Mousseau and B. Roy, ELECTRE Methods, in Multiple Criteria Decision Analysis: State of the Art Surveys, eds. J. Figueira, V. Mousseau and B. Roy (Springer, New York, 2005), pp. 131-153.

12. Y. Freund and R. E. Schapire, Experiments with a new boosting algorithm, in Proc. Int. Conf. Machine Learning (Morgan Kaufmann, San Francisco, 1996), pp. 148-156.

13. G. Demiroz and A. Guvenir, Classification by voting feature intervals, Machine Learning: ECML-97, Lecture Notes in Computer Science, Vol. 1224 (1997), pp. 85-92. 
14. P. Gupta, M. K. Mehlawat and A. Saxena, Asset portfolio optimization using fuzzy mathematical programming, Information Sciences 178(6) (2008) 1734-1755.

15. C. L. Hwang and K. Yoon, Multiple Attribute Decision Making Methods and Applications (Springer, Berlin Heidelberg, 1981).

16. M. Hall, E. Frank, G. Holmes, B. Pfahringer, P. Reutemann and I. H. Witten, The WEKA data mining software: An update, SIGKDD Explorations 11(1) (2009) $10-18$.

17. R. C. Holte, Very simple classification rules perform well on most commonly used datasets, Machine Learning 11 (1993) 63-91.

18. W. Iba and P. Langley, Induction of one-level decision trees, in Proc. Ninth Int. Conf. on Machine Learning (ML, 1992), Aberdeen, Scotland, UK, July 1-3, 1992 (Morgan Kaufmann 1992, ISBN 1-55860-247-X), pp. 233-240.

19. G. Kou, Y. Peng, Y. Shi, M. Wise and W. Xu, Discovering credit cardholders' behavior by multiple criteria linear programming, Annals of Operations Research 135(1) (2005) $261-274$.

20. G. Kou and C. Lou, Multiple factor hierarchical clustering algorithm for large scale web page and search engine clickstream data, Annals of Operations Research (2010), doi: 10.1007/s10479-010-0704-3.

21. L. I. Kuncheva, Combining Pattern Classifiers: Methods and Algorithms (Wiley, 2004).

22. Y. Kuo, T. Yang and G. W. Huang, The use of grey relational analysis in solving multiple attribute decision-making problems, Computers $\&$ Industrial Engineering 55 (2008) 80-93.

23. S. K. Lee, Y. H. Cho and S. H. Kim, Collaborative filtering with ordinal scale-based implicit ratings for mobile music recommendations, Information Sciences 180(11) (2010) 2142-2155.

24. A. S. Milani, A. Shanian and C. El-Lahham, Using different ELECTRE methods in strategic planning in the presence of human behavioral resistance, Journal of Applied Mathematics and Decision Sciences 2006 (2006) 19, doi:10.1155/JAMDS/2006/10936.

25. MATLAB, The MathWorks, Inc., Natick, MA 01760 (2005), http://www.mathworks. com/products/matlab/.

26. G. Nakhaeizadeh and A. Schnabl, Development of multi-criteria metrics for evaluation of data mining algorithms, in Proc. Third Int. Conf. on Knowledge Discovery and Data Mining (KDD'97) (Newport Beach, California, 1997), pp. 37-42.

27. D. L. Olson, Comparison of weights in TOPSIS models, Mathematical and Computer Modelling 40(7-8) (2004) 721-727.

28. S. Opricovic, Multicriteria optimization of civil engineering systems, Faculty of Civil Engineering, Belgrade (1998).

29. S. Opricovic and G. H. Tzeng, Compromise solution by MCDM methods: A comparative analysis of VIKOR and TOPSIS, European Journal of Operational Research 156(2) (2004) 445-455.

30. S. Opricovic and G. H. Tzeng, Multicriteria planning of post-earthquake sustainable reconstruction, Computer-Aided Civil and Infrastructure Engineering 17(3) (2002) $211-220$.

31. Y. Peng, G. Kou, Y. Shi and Z. Chen, A descriptive framework for the field of data mining and knowledge discovery, International Journal of Information Technology 86 Decision Making 7(4) (2008) 639-682.

32. Y. Peng, G. Kou, Y. Shi and Z. Chen, A multi-criteria convex quadratic programming model for credit data analysis, Decision Support Systems 44(4) (2008) 1016-1030. 
33. Y. Peng, G. Kou, G. Wang, W. Wu and Y. Shi, Ensemble of software defect predictors: An AHP-based evaluation method, International Journal of Information Technology $\&$ Decision Making 10(1) (2011) 187-206, doi:10.1142/S0219622011004282.

34. Y. Peng, G. Kou, G. Wang and Y. Shi, FAMCDM: A fusion approach of MCDM methods to rank multiclass classification algorithms, Omega 39(6) (2011) 677-689, doi: 10.1016/j.omega.2011.01.009.

35. Y. Peng, G. Wang and H. Wang, User preferences based software defect detection algorithms selection using MCDM, Information Sciences (2010), doi:10.1016/j. ins.2010.04.019.

36. J. R. Quinlan, C4.5: Programs for Machine Learning (Morgan Kaufmann, 1993).

37. J. Rice, The algorithm selection problem, Advances in Computers 15 (1976) 65-118.

38. B. Roy, Classement et choix en presence de points de vue multiples (la methode ELECTRE) R.I.R.O 8 (1968) 57-75.

39. B. Roy and D. Bouyssou, Aide Multicritere a la Decision: Methodes et cas (Economica, Paris, 1993).

40. B. Roy, ELECTRE III: Un algorithme de classements fonde sur une representation flue des preferences en presence de ctriteres multiples, Cahiers du CERO 20(1) (1978) 3-24.

41. L. Rokach, Ensemble-based classifiers, Artificial Intelligence Review 33(1-2) (2009) $1-39$.

42. K. A. Smith-Miles, Cross-disciplinary perspectives on meta-learning for algorithm selection, ACM Computing Surveys 41(1) (2008), Article No.: 6, doi:10.1145/ 1456650.1456656 .

43. B. Soylu, Integrating PROMETHEE II with the Tchebycheff function for multi criteria decision making, International Journal of Information Technology $\&$ Decision Making 9(4) (2010) 525-545.

44. E. Triantaphyllou and K. Baig, The impact of aggregating benefit and cost criteria in four MCDA methods, IEEE Transactions on Engineering Management 52(2) (2005) $213-226$.

45. I. H. Witten and E. Frank, Data Mining: Practical Machine Learning Tools and Techniques, 2nd edn. (Morgan Kaufmann, San Francisco, 2005).

46. G. I. Webb, Decision tree grafting from the all-tests-but-one partition, in Proc. Sixteenth Int. Joint Conf. Artificial Intelligence (IJCAI'99), Stockholm, Sweden, July 31-August 6 (1999), pp. 702-707.

47. S. M. Weiss and C. A. Kulikowski, Computer Systems that Learn: Classification and Predication Methods from Statistics, Neural Nets. Machine Learning and Expert Systems (Morgan Kaufmann, 1991).

48. J. L. Yang, H. N. Chiu and G. Tzeng, Vendor selection by integrated fuzzy MCDM techniques with independent and interdependent relationships, Information Sciences 178(21) (2008) 4166-4183.

49. Y. Ou Yang, H. Shieh, J. Leu and G. Tzeng, A Vikor-based multiple criteria decision method for improving information security risk, International Journal of Information Technology \& Decision Making 8(2) (2009) 267-287. 